\title{
Outcomes of CKD in primary care
}

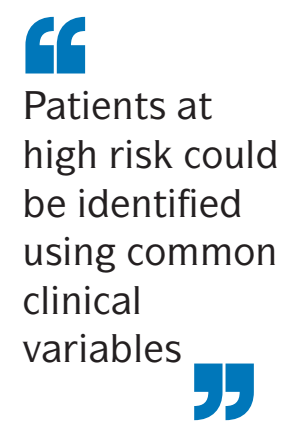

Many studies have assessed the risks associated with chronic kidney disease (CKD) but few have focused on patients managed in primary care. New findings from a 5-year study indicate that the majority of patients with stage 3 CKD in primary care demonstrate stable renal disease, with very few patients progressing to end-stage renal disease (ESRD). The researchers suggest that CKD management in primary care should focus on identifying the minority of patients at high risk of adverse outcomes, and identifying and reassuring those at low risk of disease progression.

To gain a better understanding of CKD outcomes among patients managed in primary care, Adam Shardlow and colleagues studied the outcomes of 1,741 people with stage $3 \mathrm{CKD}$ in this setting. "All participants met strict CKD diagnostic criteria, which contrasts with many previous studies that used retrospective databases and a single estimate of glomerular filtration rate to define CKD," explains Shardlow.

Over the 5-year study period, 247 participants (14.2\%) died, mostly of cardiovascular causes. Only four patients
(0.2\%) developed ESRD, and 308 (17.7\%) had evidence of CKD progression. 593 patients (34.1\%) exhibited stable CKD and 336 (19.1\%) achieved disease remission. "Patients at high risk could be identified using common clinical variables," says Shardlow. "A significant minority of participants demonstrated remission, in that they no longer had results consistent with CKD despite having previously met diagnostic criteria."

The researchers are planning a 10-year follow-up of their cohort and are investigating different approaches to estimate glomerular filtration rate in this population. "In addition, we feel that the concept of CKD remission warrants investigation in different populations, and consideration in future revisions of CKD diagnostic criteria," notes Shardlow.

Susan J. Allison

ORIGINAL ARTICLE Shardlow, A. et al. Chronic kidney disease in primary care: outcomes after five years in a prospective cohort study. PLoS Med. http://dx.doi. org/10.1371/journal.pmed.1002128 (2016) 